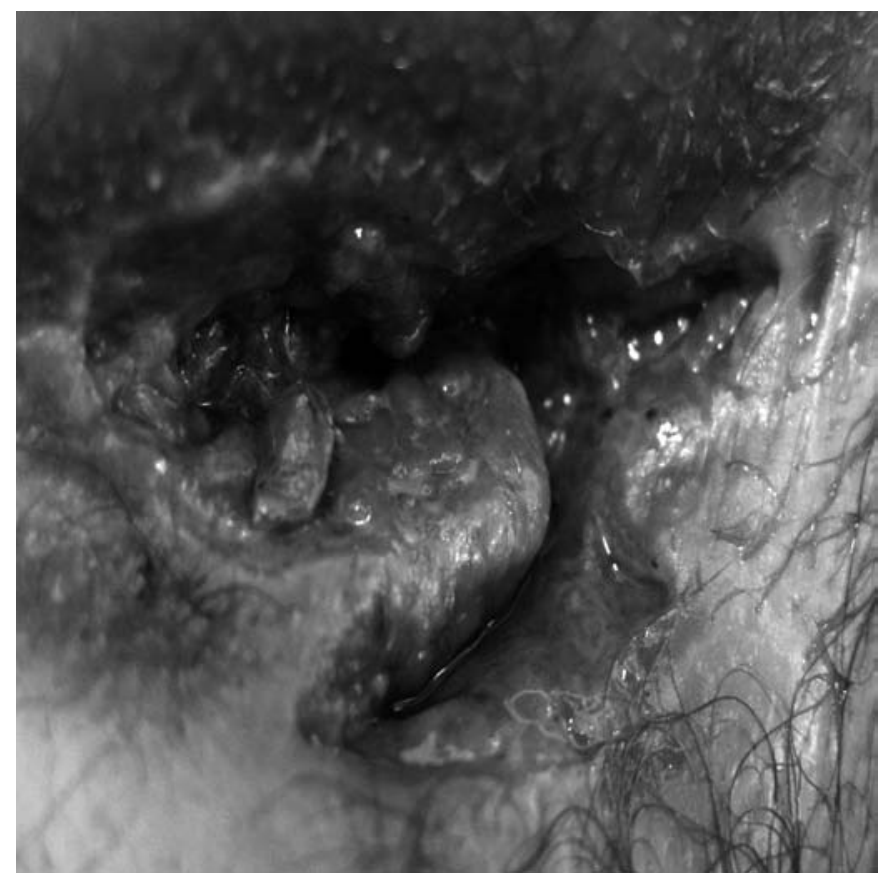

Fig. 1.

tres semanas de tratamiento oral con tuberculostáticos, las lesiones mejoraron espectacularmente quedando el paciente asintomático, sin precisar analgésicos.

Hemos presentado un caso de tuberculosis perianal (TBCPA) en un paciente sin infección por el VIH ni otros factores predisponentes para inmunodepresión. Sin la presencia concomitante de TBC pulmonar activa esta entidad es extremadamente rara habiéndose publicado pocos casos en la literatura (1). Nuestro paciente probablemente tuvo TBC pulmonar subclínica como así lo sugiere la imagen del TAC pulmonar. La afectación gastrointestinal más frecuente en el tubo digestivo se da a nivel de la válvula íleocecal, la cual en nuestro enfermo no estaba comprometida. Los casos de afectación perianal exclusiva son extremadamente raros (2). Los pocos casos descritos en la literatura de afectación perianal se han asociado a localización pulmonar primaria en dos (3) y siete descripciones (4). La forma de presentación más común es la de úlcera crónica perianal; La presentación de formas fistulizadas como en nuestro caso es aún más rara $(5,6)$. La enfermedad de Crohn juega un papel fundamental en el diagnóstico diferencial de esta entidad siendo otras alternativas la colitis ulcerosa, lesiones por Herpes simplex, Sífilis, Sarcoidosis, Amebiasis, Linfogranuloma venéreo o incluso neoplasias anales ulceradas (7). $\mathrm{La}$ biopsia en este tipo de pacientes resulta imperativa a la hora de confirmar un diagnóstico de sospecha ante una úlcera anal de larga evolución puesto que se hace muy difícil el diagnóstico diferencial macroscópicamente sobre todo entre TBCPA y Enfermedad de Crohn (8). Una de las técnicas que se ha demostrado altamente eficaz ha sido la colonoscopia con toma de biopsia de las lesiones sospechosas (9). El tratamiento se basa en la administración de fármacos tuberculostáticos durante 6-9 meses (10), con un pronóstico favorable, demostrando la recuperación completa de las lesiones como el caso de nuestro paciente a quien el tratamiento médico le ahorró una molesta intervención quirúrgica que sólo hubiera conseguido paliar su sufrimiento.

R. Oropesa Juanes, V. Abril López de Medrano, J. M. Huguet $^{1}$, J. V. Roig ${ }^{2}$, J. E. Ballester ${ }^{3}$, E. Ortega ${ }^{3}$

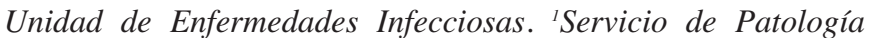
Digestiva. ${ }^{2}$ Servicio de Cirugía General. ${ }^{3}$ Unidad de Enfermedades Infecciosas. Hospital General Universitario. Valencia

1. Hardland RW, Barkey B. Anal tuberculosis report of two cases and literature review. Am J Gastroenterol 1992; 87: 1488-91.

2. Aktogu S, Yorgancioglu A, Cirak K, Kose T, Dereli SM. Clinical spectrum of pulmonary and pleural tuberculosis: A report of 5480 cases. Eur Respir J 1996; 9: 2031-5.

3. Hardland RW, Barkey B. Anal tuberculosis report of two cases and literature review. Am J Gastroenterol 1992; 87: 1488-91.

4. Sultan S, Azria S, Bauer P, Abdelnour M, Atienza P. Anoperineal tuberculosis: Diagnostic and management considerations in seven cases. Dis Colon Rectum 2002; 45: 407-10

5. Candela F, Serrano P, Arriero JM, Teruel A, Reyes D, Calpena R. Perianal disease of tuberculosis origin: Report a case and review of the literature. Dis Colon Rectum 1999; 42: 110-2.

6. Ani AN, Solanke TF. Anal fistula: A review of 82 cases. Dis Colon Rectum 1976; 19: 51-5.

7. Candela F, Serrano P, Arriero JM, Teruel A, Reyes E, Calpena R. Perianal disease of tuberculosis origin: Report a case and review of the literature. Dis Colon Rectum 1999; 42: 110-2.

8. Akgun Y. Isolated perianal tuberculosis. The Netherl J of Med 2005; 63: 115-7.

9. R Kirsch, M Pentecost, P de M Hall, D P Epstein, G Watermeyer, P W Friederich. Role of colonoscopic biopsy in distinguishing between Crohn's disease and intestinal tuberculosis. Journ of Clin Path 2006; 59: 840-4.

10. MP Sharma, Vikram Bhatia. Abdominal tuberculosis. Indian J Med Res 2004; 120: 305-15.

\section{Infarto de la arteria cerebral media como forma de presentación de la tuberculosis miliar}

\section{Sr. Director:}

A pesar del descenso mundial del número de casos de tuberculosis (TBC) pulmonar, existe en la actualidad un incremento de manifestaciones de la TBC extrapulmonar $(1,2)$, cuyo máximo exponente en términos de peor pronóstico o invalidez residual viene representado por la afectación neurológica (3). La afectación tuberculosa del SNC comprende la participación meníngea en la mayoría de las ocasiones, siendo escasas las complicaciones vasculares descritas en los pacientes adultos con ausencia de VIH (4).

Paciente de 41 años con lupus eritematoso (LES) en tratamiento con metilprednisolona, e infartos isquémicos cerebrales múltiples con paresia del VI par craneal de modo residual. Es ingresada en la UCI tras caída al suelo debido a una disminución del nivel de conciencia. A su llegada presenta un aceptable nivel de conciencia, valorado mediante la escala de Glasgow con 11 (O4V1M5), respondiendo a órdenes sencillas, aunque presentaba afasia motora, y plejia de las extremidades derechas. Otros hallazgos fueron la existencia de Raynaud bilateral y esclerodactilia. El resto de la exploración física normal.

Se realizó una TAC craneal de urgencia donde se apreció un infarto isquémico hiperagudo silviano izdo correspondiente al territorio de la arteria cerebral media (ACM) e infartos lacunares crónicos en la sustancia blanca. Así mismo se realizó un estudio con ultrasonografía mediante doppler transcraneal donde se apreciaron microembolias aisladas en todos los territorios, con un ligero aumento de la resistencia periférica en el territorio de la ACM izda.

En las diferentes exploraciones complementarias realizadas se halló una leucopenia, junto con anemia y plaquetopenia; el anticuerpo anticardiolipina fue negativo. En la Rx torax inicial aparecía un patrón micronodular con infiltrados bilaterales (Fig. 1). Se 


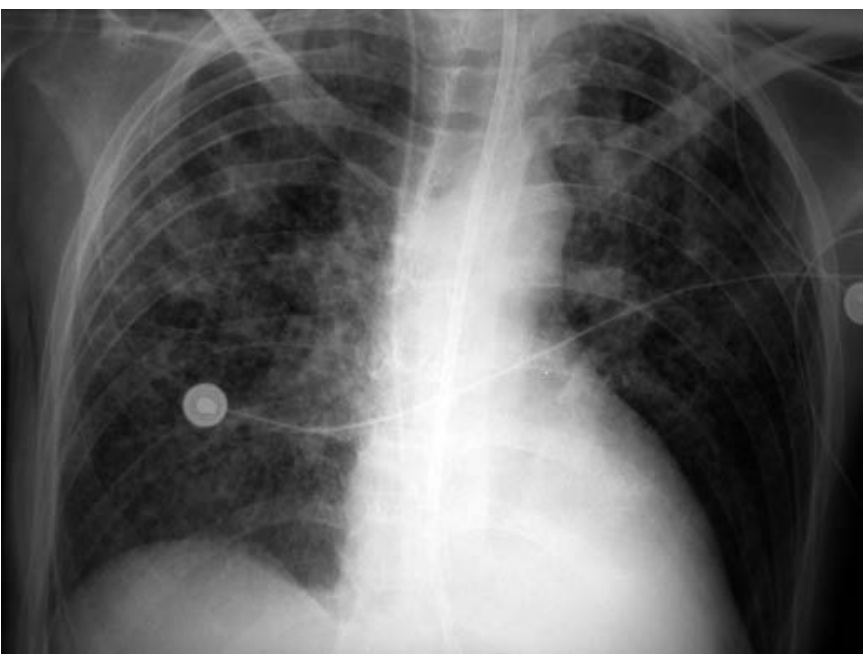

Fig. 1. Rx tórax. Patrón micronodular con infiltrados bilaterales.

extrajeron 2 baciloscopias que fueron positivas para mycobacterium tuberculosis. La ECOgrafía abdominal y el ECOcardiograma transtorácico fueron normales. Así mismo se realizó un estudio del tracto digestivo mediante la realización de una fibrogastroscopia que no mostró hallazgos patológicos y una colonoscopia donde se apreciaron unas úlceras en íleon y colon dcho sugerentes de enfermedad inflamatoria intestinal, con una biopsia de las mismas correspondientes a una ileocolitis inespecífica crónica. El cultivo del LCR mostró un abundante crecimiento de Mycobacterium tuberculosis a los 15 días de su extracción.

La evolución de la paciente inicialmente no fue desfavorable, si bien desde el punto de vista neurológico no recuperó ninguno de los déficits. Se implantó tratamiento antituberculoso con rifampicina, isoniazida, pirazinamida y etambutol, debiendo suspender el primero de los mismos por desarrollo de citolisis y colostasis. Finalmente presentó una insuficiencia respiratoria con empeoramiento radiológico con respecto al descrito previamente, por lo que precisó intubación orotraqueal y ventilación mecánica, e instauración de tratamiento con drogas vasoactivas por presentar una situación de shock refractario, que llevó a su fallecimiento.

El caso expuesto traduce la existencia de una TBC miliar, con afectación pulmonar e intestinal y desarrollo de una meningitis y un infarto isquémico de la ACM izquierda.

La TBC miliar generalmente se presenta durante los primeros 6 meses la primoinfección tuberculosa y se origina tras la erosión de un vaso sanguíneo por un foco necrótico, ocasionando una entrada masiva de bacilos en el torrente circulatorio y una diseminación de los mismos hacia numerosos órganos.

La infección meníngea por el Mycobacterium tuberculosis es la complicación neurológica más frecuente de la tuberculosis aunque se calcula que únicamente sucede en el $8 \%$ de las tuberculosis. La profilaxis y las mejoras en las condiciones sociales han disminuido la importancia de la endemia tuberculosa, salvo en poblaciones concretas como los pacientes VIH (5).

Las complicaciones del SNC comportan una elevada mortalidad y morbilidad residual $(3,6)$ y comprenden la meningoencefalitis miliar, la hidrocefalia obstructiva, la existencia de tuberculomas, así como el desarrollo de complicaciones meningovasculares como las vasculitis de arterias de mediano cabibre, que pueden ocasionar accidentes isquémicos transitorios e infartos cerebrales como en el caso expuesto (6-8).

El diagnóstico de la afectación meningea se sustenta en la demostración del bacilo en el LCR cuyo cultivo es positivo uni- camente en 1/3 de las ocasiones, por lo que ante una alta sospecha del mismo debe iniciarse el tratamiento, y el método más sensible para su diagnóstico es la determinación de la PCR en tiempo real (9).

En el presente caso a pesar de haber iniciado tratamiento de modo precoz con tuberculostáticos y corticoides $(10,11)$, la paciente falleció. Incialmente el diagnóstico de afectación meníngea secundaria a la TBC fue complicado debido a que la paciente contaba con el antecedente de lupus eritematoso que también podría originar alteraciones neurológicas frecuentes en forma de accidente cerebrovascular, aunque en parte quedó descartada la implicación de esta patología en la génesis del infarto cerebral tras la negatividad del anticuerpo antifosfolípido, principal factor causante de infarto en el LES (12). Posiblemente la intensidad de las manifestaciones extrapulmonares de la TBC en esta paciente fueron debidas al tratamiento inmunosupresor que recibía debido a su enfermedad inmune, pues existen pocos caso descritos de afectación TBC diseminada complicada con afectación del SNC (13).

\section{B. Zalba Etayo, B. Obón Azuara, I. Gutiérrez Cía, B. Villa- nueva Anadón, R. Ridruejo Sáez}

\section{Servicio de Medicina Intensiva. Hospital Clínico Universitario.} Zaragoza

1. Kourbatova EV, Borodulin BE, Borodulina EA, del Río C, Blumberg HM, Leonard MK Jr. Risk factors for mortality among adult patients with newly diagnosed tuberculosis in Samara, Russia. Int J Tuberc Lung Dis 10: 1224-30

2. Salgueiro Rodríguez M, Pérez del Molino ML, Zamarrón Sanz C, Rodríguez Suárez JR, Antúnez López J. Tuberculosis en el área de Santiago de Compostela durante los años 1999-2002. Un estudio epidemiológico. An Med Interna (Madrid) 2004; 21: 11-22.

3. Bemer P, Boutoille D, Lepelletier D, Chamoux C, Guitton C, Drugeon $\mathrm{H}$. Clinical aspects and management of patients with tuberculous meningitis. Retrospective analysis from 1994 to 2005. Rev Pneumol Clin 2006; 62: 223-9.

4. Belorgey L, Lalani I, Zakaria A. Ischemic stroke in the setting of tuberculous meningitis. J Neuroimaging 2006;16: 364-6.

5. Cowppli-Bony P, Oka DN, Datie A, Napon C, Kouassi EB.Tuberculosis and stroke: Case report and literature review. Sante 2005;15: 201-4.

6. Kourbatova EV, Leonard MK, Romero J, Kraft C, Del Río C, Blumberg HM. Risk factors for mortality among patients with extrapulmonary tuberculosis at an academic inner-city hospital in the US. Eur J Epidemiol 2006; 21: 715-21

7. Kilani B, Ammari L, Tiouiri H, Goubontini A, Kanoun F, Zouiten F, et al. Neuroradiologic manifestations of central nervous system tuberculosis in 122 adults. Rev Med Interne 2003; 24: 86-96

8. Hsieh FY, Chia LG, Shen WC. Locations of cerebral infarctions in tuberculous meningitis. Neuroradiol 1992; 34: 197-9.

9. Bhigjee AI, Padayachee R, Paruk H, et al. Diagnosis of tubercuolus meningitis: Clinical and laboratory parameters. Int J Infect Dis 2007; 22

10. Chan KH, Cheung RT, Lee R, Mak W, Ho SL. Cerebral infarcts complicating tuberculous meningitis. Cerebral infarcts complicating tuberculous meningitis. Cerebrovasc Dis 2005; 19: 391-5.

11. Thwaites GE, Macmullen-Price J, Tran TH, Pham PM, Nguyen TD Simmons CP, et al. Serial MRI to determine the effect of dexamethasone on the cerebral pathology of tuberculous meningitis: An observational study. Lancet Neurol 2007; 6: 230-6.

12. Comabella M, Tintoré M, Montalban X. Manifestaciones neuropsiquiátricas. En: J. Font, M. Khamashta y M. Vilardell eds. Lupus Eritematoso Sistémico. Ed. mra S.L. Barcelona 1996: 157-82.

13. Tsuyusaki J, Sasaki Y, Yamagishi F, Yagi T, Hashimoto T, et al. Case of disseminated tuberculosis complicated with tuberculous meningitis while investigating an abdominal lymphadenopathy. Kekkaku 2006; 81 : 667-71. 